\title{
Original Article Stature and Sex Estimation from Handprint Measurements in Population of Sharkia Governorate.
}

\author{
Somaya Tawfik Mohamed Aidy ${ }^{2}$, Wafaa Fawzy Hussein ${ }^{1}$, Aisha Abdallah Abohashem ${ }^{1}$, Bothina \\ Hassan Fouad Omran ${ }^{1}$, \\ Forensic Medicine and Clinical Toxicology Department ${ }^{1}$, Faculty of Medicine, Zagazig University \\ Forensic Medicine Authority-Ministry of Justice ${ }^{2}$, Egypt
}

\begin{abstract}
Corresponding author:
Somayatawfikaidy@gmail.co

$\mathrm{m}$

\section{ABSTRACT}

Background: At the crime scene, handprints are one of the greatest valuable evidences. Sometimes, latent impressions of hands may be the only evidence left in the crime scene. Using several anthropometric parameters on crime scene evidences as handprint for suggesting stature and sex can play an feasibility of handprint measurements in estimation of stature and sex in the age of (18 and 60 years ) from people of Sharkia governorate, stature was measured and handprints were scanned by (CanoScan lide120). Handprint parameters were processed and taken using Image $\mathrm{j}$ program measuring 21 handprint measurements. Results: As regarding the stature, showed that there is a marked difference between males and females, where males are higher in length than females in this study and all the taken measures showed noticed differences between them, Estimation of Stature from handprint measurements by equations from regression (simple linear and best fitting multiple linear models), in regard to sex estimation, all 21 handprint measurements are measurements.
\end{abstract}

\section{INTRODUCTION}

$\mathrm{H}$ uman bones play an important role for forensic anthropologist to solve criminal cases. Lately, the role of forensic anthropologist has expanded to include a wide range of forensic applications like examination of living humans in addition to human remains during natural catastrophes or in terrorism (Nagwanshi, 2019). One of the primary goals in forensic medicine is effective identification of unidentified bodies, it is cornerstone in medicolegal investigations of crime, in mass disasters and frequently in cases of death with badly preserved remains (Qura et al .,2018) .Stature is one of the main four parameters age, sex, race and stature -required to identify an individual when other evidences are corroborative (Kumar et al., 2014).Sex denotes a human biological genotype, Meanwhile
Key words: Handprint; estimation; stature; sex identifying an unknown person, sex clarification is mandatory for estimation of age and stature accurately (Krishan et al., 2016). Upon creating a biological profile, Sex identification plays a major role and ultimately adds to a hypothetical distinguishing profile of unidentified skeleton, Morphological features for sex identification has demonstrated satisfactory data for precise estimation of sex in populations and across time. It is detailed as the foremost commonly utilized technique in forensic examination (Colman et al., 2018). There are many anthropometric approaches reported to assess stature from diverse body parts, as head measurements and bones of arms and legs measurements. Many reports examined stature from hand measurements either straight forwardly from actual hands, or from hand bones, For that 
reason foot and handprint were utilized, More focus was directed to Footprint for estimation of stature more than that of handprint, Only a few works were done in the handprint

(Paulis, 2015).Using several anthropometric parameters on crime scene evidences as handprint for suggesting stature and sex can play an important role in decreasing number of accused people then can be confirmed by the usual methods like (DNA) because handprint frequently recorded at crime scenes (Komar and Buikstra., 2008). Many researches done on sex estimation with a high degree of precision from anthropometric analysis of the hand. However, most of previous reports have been focused on a small number of populations (Ishak et al., 2012). The aim of the present study was to determine the feasibility of handprint measurements in stature and sex estimation from handprints in our local population of Sharkia Governorate, as the use of the handprint measurements for stature and sex estimation is a new method in forensic and medicolegal studies.

\section{MATERIALS, SUBJECTS AND METHOD}

\subsection{Subjects:}

The analyzed study sample consists of 127 male and female participants lived in Sharkia governorate. The sample included 62 male individuals (the range of their age was 25-50 years) and 65 female individuals (the range of their age was 18-60 years).There were insignificant differences between right and left hand measures as shown in several studies so in this study, only right hands of right handed persons were included (Sanli et al.,2005 and Habib and Kamal,2010 ) with exclusion of any musculoskeletal deformities or skin diseases.

\subsection{Methods:}

\section{Study design:}

This study is a cross-sectional study which was done in Department of Forensic Medicine and Clinical Toxicology, Zagazig University and Zagazig Authority Forensic Medicine - 1st of July 2018 to 30th of June 2019. Institution Review Board (IRB) of Zagazig University faculty of Medicine, had approved the study protocol, participants informed and written consent obtained from each participant

Exclusion criteria were:

Age: below eighteen years old and more than sixty years old. Any musculoskeletal deformities and any skin diseases.

\subsection{Tools and instruments:}

Flatbed scanner (CanoScan lide 120) (Fig.1) with a ruler to take images of the hand at three hundreds dots per inch (paulis, 2015) and stadiometer for living stature.

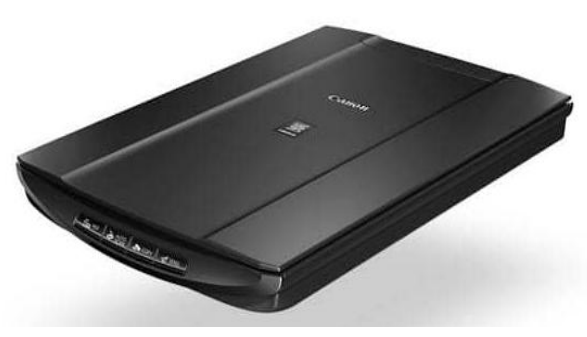

(Figure 1): Canoscan lide 120

\section{Steps of performance and techniques used:}

1-Living length (stature) estimation:

Stature was measured according to Paulis (2015) in which each participant was informed to stand bare foots with on stadiometer flat surface with attached heels together, both hand in the side of the thighs, the head was in the horizontal plane against the vertical board (The stadiometer movable rod put in contact with the vertex in the middle sagittal plane). The measurements were repeated and the mean measures were documented (by one observer) to avoid inter-observer variations. All measurements were measured in centimeters to the nearest millimeters (Fig. 2). 


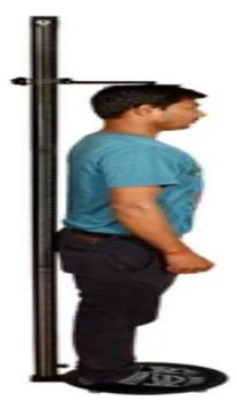

(Figure 2): Stadiometer

2-Handprint acquisition:

By using flatbed scanner (CanoScan lide120) and a scale, the subject was requested to put his / her right hand on the scanner without pressure where the thumb was in a right angle with the medially adducted fully expanded four fingers.

Handprint measurements were taken by a specific program in distance measurement in images called ImageJ $1.52 \mathrm{~K}$ measurement. A scale was used in the scanner during handprint capturing to measure the length. The program save the data in excel file to decrease the error of manual manipulation of the data.

\section{3- Handprint measurements:}

Using distance in images measuring program, the following measures was taken according to (Ishak et al., 2012 and Paulis, 2015).

Phalangeal length:

Proximal and middle phalangeal length were measured as the distance between the centers of the phalangeal creases, while the distal phalangeal length was obtained by the distance between the most forwarding projecting point on the tip of a finger to the center of first distal phalange crease (Fig. 3).

\section{Handprint breadth (HPB):}

It was represented as the distance between the most pointed part of the palm print on lateral side at the level of the second metacarpal to the most pointed part on medial side of the palm print at the distal transverse crease (Figure 4).

\section{Handprint length (HPL):}

It was represented as the distance from the center of the base of the print to the most projecting part at the tip of the middle finger (Fig. 4).

Finerprint length:

It was measured as distance between the centers of the proximal flexion crease of the finger to the tip of the finger (Fig. 4).

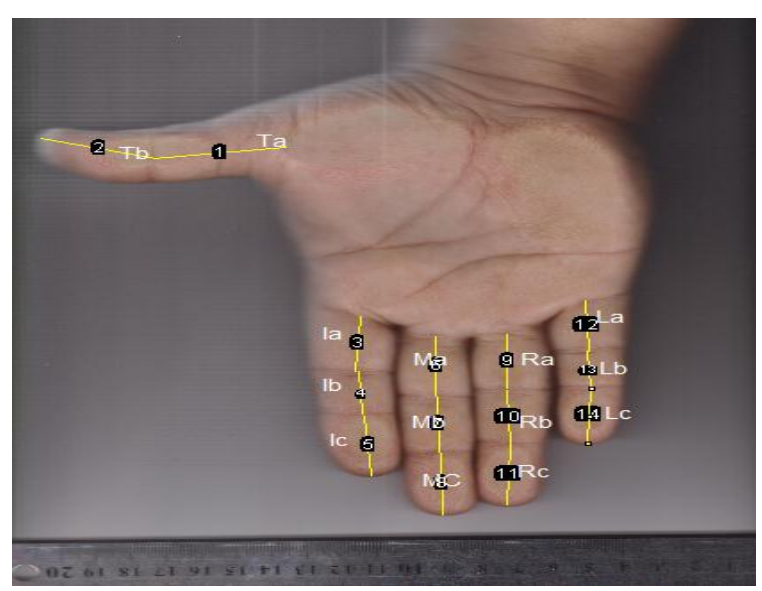

(Figure 3): Phalangeal print measurements.

Ta: Proximal and Tb: distal phalange print length of the thumb

Ia: Proximal, Ib: middle and Ic: distal phalange print length of index finger

Ma: Proximal, Mb: middle and Mc: distal phalange print length of middle finger

$\mathrm{Ra}$ : Proximal, Rb: middle and Rc: distal phalange print length of ring finger

La Proximal, Lb middle and Lc distal phalange print length of little finger 


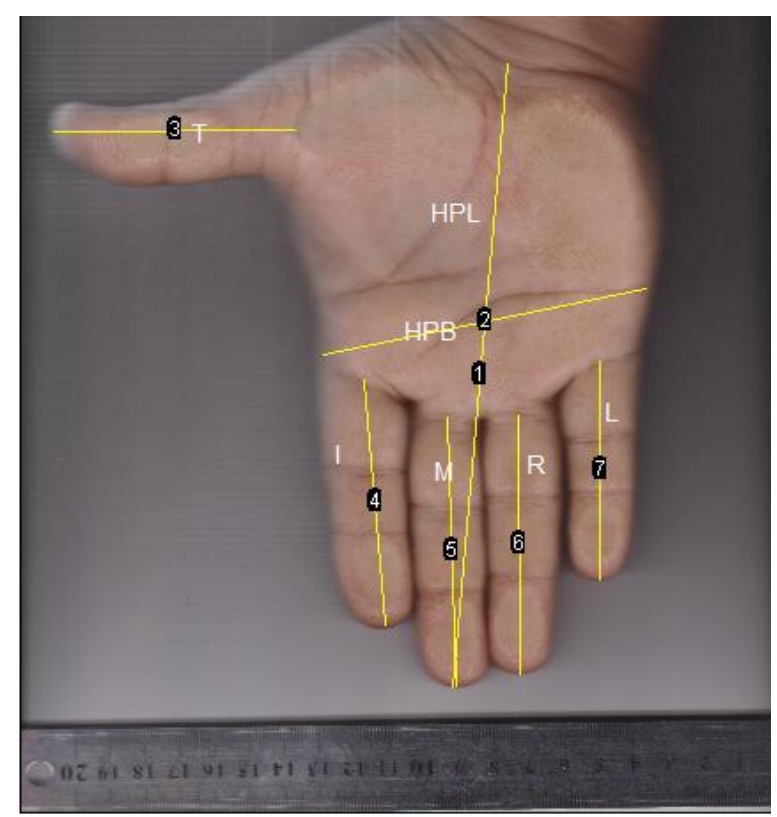

(Figure 4): Handprint measurements.

HPL: Handprint length

HPB: Handprint breadth
$\mathrm{T}$ : Thumb finger
$\mathrm{M}$ : Middle finger
$\mathrm{R}$ : Ring finger
I: Index finger

\subsection{Statistical analysis:}

All data were gathered, represented in tables and statistically tested using SPSS 20.0 for windows (SPSS Inc., Chicago,

IL, USA). The data were expressed as the mean \pm SD and median. Qualitative data were represented as absolute frequencies (number) and relative frequencies (percentage). Continuous data were examined for normality by using Shapiro Walk test (Ghasemi and zahediasl, 2012).

1) Independent samples Student's t-test was used to compare between two groups of normally distributed variables.

2) Pearson's correlation coefficient was calculated, (+) sign indicate direct correlation \& (-) sign indicate inverse correlation, "p-value $<0.05 "$ was considered statistically significant (S), "pvalue < 0.001" was considered highly significant (HS), and "p-value $\geq 0.05$ " was statistically insignificant (NS).

3) $P$ (regression model): assesses the statistical significance of each independent variable included in the model. The model for simple linear regression, given $\mathrm{n}$ observations, is:

"Y $=\mathrm{a}+\beta 1 \mathrm{X} 1 "$

"Y= the variable that we are trying to predict"

"X = the variable that are using to predict"

" $\mathrm{a}=$ the intercept (Constant)"

$" \beta=$ coefficient of $x$ (The mean change in the dependent variable) for one unit of change in the predictor variable, while holding other predictors in the model constant)" (Reel et al., 2012).

R2 (coefficient of determination): is the proportion of the variance in the dependent variable that is predictable from the independent variable(s)

$\mathrm{T}$ test $=$ test of significant

4) Multiple regressions: Situations frequently occur in which we are interested in the dependency of a dependent variable on several independent variables. The model for multiple linear regressions, given $n$ observations, is

" $Y=a+\beta 1 X 1+\beta 2 X 2+\beta 3 X 3+\ldots \ldots . "$

" $\mathrm{Y}=$ the variable that we are trying to predict"

" $\mathrm{X}=$ the variable that are using to predict "

$\mathrm{a}=$ the intercept (Constant)"

$" \beta=$ coefficient of $x$ (The mean change in the dependent variable for one unit of change in the predictor variable, while holding other predictors in the model constant)" (Reel et al., 2012).

T test =test of significant

5) Accuracy: equal summation of true positive+ true negative divide to all studied patients $=\{\mathrm{a}+\mathrm{d} / \mathrm{a}+\mathrm{b}+\mathrm{c}+\mathrm{d})\}^{*}$ 100

6) Wilk's lambda: It is a test of significance for discriminant function analysis. Its value ranges between 0 and 1. The nearer the value is to zero; the 
more important the model is in differentiating between males and females.

\section{RESULTS}

I) Demographic data of the studied group:

127 participants, were included in this study , $62(48.8 \%)$ males had mean of age ( $39.4 \pm 11.4$ )years , 65 females $(51.2 \%)$ with mean age of $40.6 \pm 10.5$ years with lower range is 18 years old while the upper range was 60 years as shown in (Table 1). 
Table (1): Demographic criteria of the study population:

\begin{tabular}{|l|l|l|l|}
\hline & Male & Female & Total \\
\hline $\begin{array}{l}\text { Num. } \\
\text { (Percentage \%) }\end{array}$ & 62 & 65 & 127 \\
$(48.8 \%)$ & $(51.2 \%)$ & $(100 \%)$ \\
\hline $\begin{array}{l}\text { Age (18-60) } \\
\text { mean } \pm \text { SD) }\end{array}$ & $39.4 \pm 11.4$ & $40.6 \pm 10.5$ & $40 \pm 11$ \\
\hline
\end{tabular}

SD: standard deviation

\section{II) Stature estimation from handprint and phalangeal measurements:}

1) Comparison between males and females regarding stature, hand and phalangeal print parameters:

Using student -t- test, there were significant recorded differences between male and female measures, where males were larger in length than females, mean stature of males was
$172.7 \pm 7.1 \mathrm{~cm}$ while in females was $160.9 \pm$ $6.3 \mathrm{~cm}$. All the measures expressed differences between male and female significantly ( $\mathrm{p}$ value $<0.05$ ). Also, all males' handprint measurements were larger than the equivalent females (Tables 2).

Table (2): Comparison between males and females regarding stature, hand and phalangeal print parameters (student -t- test):

\begin{tabular}{|c|c|c|c|c|}
\hline \multirow[t]{2}{*}{ Variables } & \multicolumn{2}{|c|}{ Studied group } & \multirow[t]{2}{*}{$\mathbf{t}$} & \multirow[t]{2}{*}{$\mathbf{p}$} \\
\hline & $\begin{array}{l}\text { Male }=62 \\
\text { mean } \pm\end{array}$ & $\begin{array}{l}\text { Female }=65 \\
\text { mean } \pm\end{array}$ & & \\
\hline Stature & $172.7 \pm 7.1$ & $160.9 \pm 6.3$ & 9.9 & $<0.001(\mathrm{~S})$ \\
\hline Handprint length & $19.1 \pm 1.2$ & $17.4 \pm 0.88$ & 9.4 & $<0.001(\mathrm{~S})$ \\
\hline Handprint & $9.135 \pm 0.56$ & $8.2 \pm 0.5$ & 9.9 & $<0.001(\mathrm{~S})$ \\
\hline Thumb finger & $6.78 \pm 0.54$ & $6.2 \pm 0.4$ & 7.2 & $<0.001(\mathrm{~S})$ \\
\hline Index finger & $7.38 \pm 0.54$ & $6.78 \pm 0.62$ & 5.8 & $<0.001(\mathrm{~S})$ \\
\hline Middle finger & $8.2 \pm 0.57$ & $7.54 \pm 0.47$ & 7.1 & $<0.001(\mathrm{~S})$ \\
\hline Ring finger & $7.7 \pm 0.56$ & $7 \pm 0.47$ & 7.9 & $<0.001(\mathrm{~S})$ \\
\hline Little finger & $6.2 \pm 0.62$ & $5.65 \pm 0.43$ & 5.8 & $<0.001(\mathrm{~S})$ \\
\hline Thumb a & $3.42 \pm 0.38$ & $3.2 \pm 0.43$ & 3.1 & $<0.003(\mathrm{~S})$ \\
\hline Thumb b & $3.5 \pm 0.27$ & $3.05 \pm 0.22$ & 10 & $<0.001(\mathrm{~S})$ \\
\hline Index a & $2.54 \pm 0.32$ & $2.38 \pm 0.29$ & 2.9 & $<0.004(\mathrm{~S})$ \\
\hline Index $b$ & $2.27 \pm 0.2$ & $2.12 \pm 0.22$ & 4 & $<0.001(S)$ \\
\hline Index c & $2.63 \pm 0.22$ & $2.4 \pm 0.18$ & 6.3 & $<0.001(\mathrm{~S})$ \\
\hline Middle a & $2.87 \pm 0.32$ & $2.63 \pm 0.27$ & 4.7 & $<0.001(S)$ \\
\hline
\end{tabular}


Stature and sex estimation from hand.......

\begin{tabular}{|l|l|l|l|l|}
\hline Middle b & $2.6 \pm 0.27$ & $2.4 \pm 0.23$ & 3.7 & $<0.001(\mathrm{~S})$ \\
\hline Middle c & $2.73 \pm 0.21$ & $2.48 \pm 0.16$ & 7.3 & $<0.001(\mathrm{~S})$ \\
\hline Ring a & $2.5 \pm 0.27$ & $2.3 \pm 0.24$ & 5.7 & $<0.001(\mathrm{~S})$ \\
\hline Ring b & $2.4 \pm 0.27$ & $2.25 \pm 0.23$ & 4 & $<0.001(\mathrm{~S})$ \\
\hline Ring c & $2.7 \pm 0.244$ & $2.46 \pm 0.18$ & 6.8 & $<0.001(\mathrm{~S})$ \\
\hline Little a & $2 \pm 0.29$ & $1.85 \pm 0.24$ & 2.8 & $0.005(\mathrm{~S})$ \\
\hline Little b & $1.8 \pm 0.19$ & $1.63 \pm 0.33$ & 4.3 & $<0.001(\mathrm{~S})$ \\
\hline Little c & $2.5 \pm 0.23$ & $2.29 \pm 0.16$ & 6.4 & $<0.001(\mathrm{~S})$ \\
\hline
\end{tabular}

\section{(t): student -t-Test $\quad$ S: significant $\quad$ P value $<0.05$}

2) Correlation matrix of participants' stature with hand and phalangeal print parameters:

Significant correlation (positive) between stature and all handprint measurements $(\mathrm{p}<0.05)$ as described in Table (3)

Table (3): Correlation matrix of participants' stature and handprint parameters $(n=127)$ :

\begin{tabular}{|l|l|l|}
\hline \multirow{2}{*}{ Variables } & Stature(N=127) & $\mathrm{p}$ \\
\cline { 2 - 3 } & $(\mathrm{r})$ & $<0.001(\mathrm{~S})$ \\
\hline Handprint length & 0.706 & $<0.001(\mathrm{~S})$ \\
\hline Handprint breadth & 0.537 & $<0.001(\mathrm{~S})$ \\
\hline Thumb finger & 0.589 & $<0.001(\mathrm{~S})$ \\
\hline Index finger & 0.541 & $<0.001(\mathrm{~S})$ \\
\hline Middle finger & 0.616 & $<0.001(\mathrm{~S})$ \\
\hline Ring finger & 0.601 & $<0.001(\mathrm{~S})$ \\
\hline Little finger & 0.465 & $<0.001(\mathrm{~S})$ \\
\hline Thumb a & 0.342 & $<0.001(\mathrm{~S})$ \\
\hline Thumb b & 0.644 & $<0.001(\mathrm{~S})$ \\
\hline Index a & 0.358 & $<0.001(\mathrm{~S})$ \\
\hline Index b & 0.399 & $<0.001(\mathrm{~S})$ \\
\hline Index c & 0.543 & $<0.001(\mathrm{~S})$ \\
\hline Middle a & 0.508 & $<0.001(\mathrm{~S})$ \\
\hline Middle b & 0.483 & $<0.001(\mathrm{~S})$ \\
\hline Middle c & 0.506 & $<0.001(\mathrm{~S})$ \\
\hline Ring a & 0.478 & $<0.001(\mathrm{~S})$ \\
\hline Ring b & 0.341 & $<0.001(\mathrm{~S})$ \\
\hline Ring c & 0.401 & $<0.001(\mathrm{~S})$ \\
\hline Little a & 0.280 & $<0.001(\mathrm{~S})$ \\
\hline Little b & 0.382 & $<0.001$ \\
\hline Little c & $0.464 * *$ & \\
\hline & & $\mathrm{P}$ \\
\hline
\end{tabular}

$(\mathrm{S})$ : significant

$\mathrm{P}$ value $<0.05$

r: correlation coefficient 
3) Regression (Simple linear model) for prediction of stature from handprint and phalangeal print measures : by equations derived from regression(simple linear), stature can be estimated by all handprint and phalangeal print parameters that were highest $\mathrm{R}^{2}$ (coefficient of determination) are with handprint Length (0.499), middle finger $(0.380)$, ring finger $(0.362)$ beside thumb b (0.415), index $\mathrm{c}(0.295)$ and middle a (0.258) illustrated by Table (4).

Table (4): regression (Simple linear model) for prediction of stature from handprint and phalangeal print measures:

\begin{tabular}{|l|l|l|l|l|}
\hline $\mathrm{N}=127$ & $\mathrm{R}^{2}$ & Equation detect stature & $\mathrm{T}$ & $\mathrm{p}$ \\
\hline Handprint length & 0.499 & $81.3+4.69^{*}$ handprint length & 11.150 & $<0.0001(\mathrm{~S})$ \\
\hline Handprint breadth & 0.288 & $107.4+6.847^{*}$ handprint breadth & 7.119 & $<0.0001(\mathrm{~S})$ \\
\hline Thumb finger & 0.347 & $107.5+19.16^{*}$ thumb & 8.158 & $<0.0001(\mathrm{~S})$ \\
\hline Index finger & 0.292 & $114.6+7.36 *$ index & 7.186 & $<0.0001(\mathrm{~S})$ \\
\hline Middle finger & 0.380 & $96.3+8.954^{*}$ middle & 8.751 & $<0.0001(\mathrm{~S})$ \\
\hline Ring finger & 0.362 & $104+8.55^{*}$ ring & 8.416 & $<0.0001(\mathrm{~S})$ \\
\hline Little finger & 0.217 & $125.5+6.96 *$ little & 5.878 & $<0.0001(\mathrm{~S})$ \\
\hline Thumb a & 0.117 & $142.7+7.277 *$ thumb a & 4.075 & $<0.001(\mathrm{~S})$ \\
\hline Thumb b & 0.415 & $110+17.322^{*}$ thumb b & 9.409 & $<0.001(\mathrm{~S})$ \\
\hline Index a & 0.128 & $141+10.267 *$ index a & 4.286 & $<0.001(\mathrm{~S})$ \\
\hline Index b & 0.159 & $131.6+16.005^{*}$ index b & 4.859 & $<0.001(\mathrm{~S})$ \\
\hline Index c & 0.295 & $114+20.944 *$ index c & 7.228 & $<0.001(\mathrm{~S})$ \\
\hline Middle a & 0.258 & $127.9+14.133^{*}$ middle a & 6.585 & $<0.001(\mathrm{~S})$ \\
\hline Middle b & 0.233 & $127.9+16.637 *$ middle b & 6.164 & $<0.001(\mathrm{~S})$ \\
\hline Middle c & 0.256 & $113.7+20.36 * 9$ middle c & 6.567 & $<0.001(\mathrm{~S})$ \\
\hline Ring a & 0.229 & $130.9+14.744^{*}$ ring a & 6.087 & $<0.001(\mathrm{~S})$ \\
\hline Ring b & 0.116 & $139.6+11.635^{*}$ ring b & 4.056 & $<0.001(\mathrm{~S})$ \\
\hline Ring c & 0.161 & $129.7+14.309^{*}$ ring c & 4.894 & $<0.001(\mathrm{~S})$ \\
\hline Little a & 0.079 & $148.9+9.289 *$ little a & 3.264 & $<0.001(\mathrm{~S})$ \\
\hline Little b & 0.146 & $140.9+15.133^{*}$ little b & 4.624 & $<0.001(\mathrm{~S})$ \\
\hline Little c & 0.215 & $123+18.147 *$ little c & 5.8 & $<0.001(\mathrm{~S})$ \\
\hline
\end{tabular}

(S): significant $\quad \mathrm{t}$ : student -t-Test $\quad \mathrm{P}$ value $<0.05 \quad \mathrm{R}^{2:}$ coefficient of determination

3) Best fitting multiple linear regression models for prediction of stature from hand and phalangeal print parameters:

Regarding the stature, using best fitting multiple linear regression model for predicting stature with variables entered and excluded, the only statistically significant independent predictor was handprint length and ring $\mathrm{b}$. The model explains $52 \%$ of stature as described in Table (5).

Table (5): Best fitting multiple linear regression models for prediction of stature from hand and phalangeal print parameters:

\begin{tabular}{|l|l|l|l|l|}
\hline \multirow{2}{*}{} & \multicolumn{2}{|l|}{$\begin{array}{l}\text { Unstandardized } \\
\text { Coefficients }\end{array}$} & P \\
\cline { 2 - 3 } & B & Std. Error & & \\
\hline Constant & 81.7 & & 10.207 & $<0.001(\mathrm{~S})$ \\
\hline $\begin{array}{l}\text { Handprint } \\
\text { Length }\end{array}$ & 5.498 & 0.539 & 2.338 & $<0.05(\mathrm{~S})$ \\
\hline Ring B & 6.468 & 2.766 & & \\
\hline
\end{tabular}

B: coefficient of regression Model ANOVA: $F=67$
Std.Error: standard error

(S): significant
$\mathrm{R}^{2}$ coefficient of determination $=0.52$ 
III) Sex estimation from handprint and phalangeal measurements:

1)Cut off level of stature, hand and phalangeal print parameters with its accuracy to identify males and females:

Demarcation points which are measured using the respective mean of the male and female measurement are described in tables (6), the calculated value represent the threshold above which an individual is classified as male and vice versa to be classified as female. Jackknifed estimated classification accuracy rates and sex-biases are provided; only those functions that classify above $80 \%$ accuracy .As shown by the univariate comparisons, the most dimorphic individual measurements are those that yielded the highest expected sex classification accuracy which were stature $84.3 \%$, HPB $84.3 \%$ and thumb b $81 \%$.

Table (6): Cut off level of stature, hand and phalangeal print parameters with its accuracy to identify males and females:

\begin{tabular}{|c|c|c|c|c|c|}
\hline \multirow[t]{2}{*}{ Variables } & \multirow{2}{*}{$\begin{array}{l}\text { Cut off } \\
\text { value } \\
\mathrm{cm}\end{array}$} & \multicolumn{2}{|c|}{ Studied group } & \multirow{2}{*}{$\begin{array}{c}\text { True } \\
\text { diagnose } \\
\text { cases }\end{array}$} & \multirow[b]{2}{*}{ Accuracy\% } \\
\hline & & $\operatorname{Male}(n=62)$ & $\begin{array}{c}\text { Female } \\
(\mathrm{n}=65)\end{array}$ & & \\
\hline Stature & $\begin{array}{l}\geq 163.5 \\
<163.5\end{array}$ & $\begin{array}{c}60 \\
2\end{array}$ & $\begin{array}{l}18 \\
47\end{array}$ & $107(60+47)$ & 84.3 \\
\hline $\begin{array}{l}\text { Handprint } \\
\text { Length }\end{array}$ & $\begin{array}{l}\geq 17.6750 \\
<17.6750\end{array}$ & $\begin{array}{c}56 \\
6\end{array}$ & $\begin{array}{l}25 \\
40\end{array}$ & $96(56+40)$ & 75.6 \\
\hline $\begin{array}{l}\text { Handprint } \\
\text { Breadth }\end{array}$ & $\begin{array}{l}\geq 8.550 \\
<8.550\end{array}$ & $\begin{array}{c}54 \\
8\end{array}$ & $\begin{array}{l}12 \\
53\end{array}$ & $107(54+53)$ & 84.3 \\
\hline Thumb & $\begin{array}{l}\geq 6.45 \\
<6.45\end{array}$ & $\begin{array}{l}49 \\
13\end{array}$ & $\begin{array}{l}19 \\
46\end{array}$ & $95(49+46)$ & 74.8 \\
\hline Index & $\begin{array}{l}\geq 7.050 \\
<7.050\end{array}$ & $\begin{array}{l}48 \\
14\end{array}$ & $\begin{array}{l}18 \\
47\end{array}$ & $95(48+47)$ & 74.8 \\
\hline Middle & $\begin{array}{l}\geq 7.6502 \\
<7.650\end{array}$ & $\begin{array}{c}54 \\
8\end{array}$ & $\begin{array}{l}25 \\
40\end{array}$ & $95(54+40)$ & 74.8 \\
\hline Ring & $\begin{array}{l}\geq 7.250 \\
<7.250\end{array}$ & $\begin{array}{l}51 \\
11\end{array}$ & $\begin{array}{l}16 \\
49\end{array}$ & $100(51+49)$ & 79.5 \\
\hline Little & $\begin{array}{l}\geq 5.750 \\
<5.750\end{array}$ & $\begin{array}{l}49 \\
13\end{array}$ & $\begin{array}{l}23 \\
42\end{array}$ & $91(49+42))$ & 71.6 \\
\hline
\end{tabular}




\begin{tabular}{|c|c|c|c|c|c|}
\hline Thumb a & $\begin{array}{l}\geq 3.25 \\
<3.25\end{array}$ & $\begin{array}{l}46 \\
16\end{array}$ & $\begin{array}{l}23 \\
42\end{array}$ & $88(46+42)$ & 69 \\
\hline Thumb b & $\begin{array}{l}\geq 3.25 \\
<3.25\end{array}$ & $\begin{array}{l}50 \\
12\end{array}$ & $\begin{array}{l}12 \\
53\end{array}$ & $103(50+53)$ & 81 \\
\hline Index a & $\begin{array}{l}\geq 2.25 \\
<2.25\end{array}$ & $\begin{array}{c}54 \\
8\end{array}$ & $\begin{array}{l}41 \\
24\end{array}$ & $78(54+24)$ & 61.4 \\
\hline Index b & $\begin{array}{l}\geq 2.15 \\
<2.55\end{array}$ & $\begin{array}{l}46 \\
16\end{array}$ & $\begin{array}{l}30 \\
35\end{array}$ & $81(46+35)$ & 63.8 \\
\hline Index C & $\begin{array}{l}\geq 2.55 \\
<2.55\end{array}$ & $\begin{array}{l}39 \\
23\end{array}$ & $\begin{array}{c}9 \\
56\end{array}$ & $95(39+56)$ & 74.8 \\
\hline Middle a & $\begin{array}{l}\geq 2.75 \\
<2.75\end{array}$ & $\begin{array}{l}45 \\
17\end{array}$ & $\begin{array}{l}18 \\
47\end{array}$ & $92(45+47)$ & 72.4 \\
\hline Middle b & $\begin{array}{l}\geq 2.45 \\
<2.45\end{array}$ & $\begin{array}{l}46 \\
16\end{array}$ & $\begin{array}{l}32 \\
33\end{array}$ & $79(46+33)$ & 62 \\
\hline Middle c & $\begin{array}{l}\geq 2.55 \\
<255\end{array}$ & $\begin{array}{l}49 \\
13\end{array}$ & $\begin{array}{l}18 \\
47\end{array}$ & $96(49+47)$ & 75.6 \\
\hline Ring a & $\begin{array}{l}\geq 2.45 \\
<2.45\end{array}$ & $\begin{array}{l}40 \\
22\end{array}$ & $\begin{array}{l}16 \\
49\end{array}$ & $89(40+49)$ & 70 \\
\hline Ring $b$ & $\begin{array}{l}\geq 2.25 \\
<2.25\end{array}$ & $\begin{array}{l}51 \\
11\end{array}$ & $\begin{array}{l}32 \\
33\end{array}$ & $84(51+33)$ & 66 \\
\hline Ring c & $\begin{array}{l}\geq 2.55 \\
<2.55\end{array}$ & $\begin{array}{l}47 \\
15\end{array}$ & $\begin{array}{l}19 \\
46\end{array}$ & $93(47+46)$ & 73.2 \\
\hline Little a & $\begin{array}{l}\geq 1.95 \\
<1.95\end{array}$ & $\begin{array}{l}37 \\
25\end{array}$ & $\begin{array}{l}21 \\
44\end{array}$ & $81(37+44)$ & 63.8 \\
\hline Little b & $\begin{array}{l}\geq 1.65 \\
<1.65\end{array}$ & $\begin{array}{l}49 \\
13\end{array}$ & $\begin{array}{l}30 \\
35\end{array}$ & $84(49+35)$ & 66 \\
\hline Little c & $\begin{array}{l}\geq 2.45 \\
<2.45\end{array}$ & $\begin{array}{l}41 \\
21\end{array}$ & $\begin{array}{l}13 \\
52\end{array}$ & $93(41+52)$ & 73.2 \\
\hline
\end{tabular}

Accuracy: true detected males +true detect females / total studied* 100

2)Stepwise discriminant analysis of hand print parameters to detect sex:

In the stepwise discriminant analysis of the handprint measures as shown in Table (7), two measurements were selected: handprint breadth and thumb b; accuracy of sex classification after cross-validation was $86.6 \%$ with bias of sex $-0.8 \%$. 
Table (7): Stepwise discriminant analysis of handprint parameters to detect sex:

\begin{tabular}{|c|c|c|c|c|c|c|c|}
\hline & \multicolumn{2}{|c|}{$\begin{array}{l}\text { Canonical Discriminant Function } \\
\text { Coefficients }\end{array}$} & \multirow[b]{2}{*}{$\begin{array}{l}\text { Wilks' } \\
\text { Lambda }\end{array}$} & \multirow[b]{2}{*}{$\begin{array}{l}\text { Functions at } \\
\text { Group } \\
\text { Centroids }\end{array}$} & \multirow[b]{2}{*}{$\begin{array}{l}\text { Section } \\
\text { level }\end{array}$} & \multirow[b]{2}{*}{ Accuracy } & \multirow[b]{2}{*}{$\begin{array}{l}\text { Sex } \\
\text { bias }\end{array}$} \\
\hline & Unstandardized & Standardized & & & & & \\
\hline Thumb b & 2.458 & 0.612 & 0.555 & $\mathrm{~F}=1.062$ & 0.0255 & $86.6 \%$ & -0.8 \\
\hline $\begin{array}{l}\text { Handprint } \\
\text { breadth }\end{array}$ & 1.163 & 0.611 & 0.454 & $\mathrm{M}=1.113$ & & & $\%$ \\
\hline constant & -18.096 & & & & & & \\
\hline
\end{tabular}

Equation for determination sex from hand print parameters:

Sex can be predicted from this equation:

$$
\text { Sex }=-18.096+1.163 * \text { Hand breadth }
$$
$+2.458 *$ Thumb b.

If the result above or equal to 0.0255 this predicts that the handprint belongs to male and below 0.0255 predicts that the handprint belongs to female with accuracy $86.6 \%$.
3) Posterior probability of hand print to detect sex :

Posterior probability percentages of exact sex arrangement are shown in Table (8) and it is evident those males were correctly distinguished at above $88.6 \%$ certainty and that females were correctly distinguished at above $85 \%$.

Table (8): posterior probability of hand print to detect sex:

\begin{tabular}{|l|c|c|c|c|c|}
\hline \multirow{2}{*}{} & \multicolumn{2}{|c|}{ Sex } & \multicolumn{2}{c|}{ posterior probability } \\
\cline { 2 - 6 } Variables & Male No/ (\%) & Female No/ (\%) & Accuracy & Male & Female \\
\hline $\begin{array}{l}\text { Stepwise } \\
\text { discriminant }\end{array}$ & $52(84 \%)$ & $58(89 \%)$ & $110(86.6 \%)$ & $88.6 \%$ & $85 \%$ \\
\hline
\end{tabular}

\section{DISCUSSION}

In issue of suspects' identification, for over a decade, law enforcements agencies all over the world used handprints for comparing the hand prints from crime scenes and the suspects, while in absence of suspects it is upcoming question if the hand print a ., 2012), the current study aims to answer this question. In the past few years, many studies were conducted on handprint measurement. Abdel-Malek et al. (1990) reported that in Egyptians, stature could be estimated from 
frand measurements, followect by Jasuja and Singh (2004) that calculated the hand length and breadth of impression of the hand by ink, also Ahemad and Purkait (2011) used impression of male subjects. Lastly, to escape staining hand by ink, Ishak et al.,(2012) and Paulis (2015) as in our conducted research, the usual measurement were taken by computerized software of scanned images of right hand to take 21 hand and phalangeal dimensions.

In the current research, there was a marked difference among males and females, Men are taller than women. Those differences may be explained by the earlier maturation of girls before boys hence boys have more physical growth by two years (Krishan and Sharma, 2007). In concordance with studies in similar population like (Habib and Kamal, 2010 and Aboul-Hagag et al., 2011), and other populations like (Jasuja and Singh, 2004; Tang et al., 2012 and Ozaslan et al.,2012) found differences that was significant statistically between males and females.

On comparison with other studies done in Egypt, the mean of the stature in our study was $(172.7 \pm 7.1 \mathrm{~cm}$ for males and $160.9 \pm$ $6.3 \mathrm{~cm}$ for females) was smaller than that of Habib and Kamal (2010) regarding males and slightly larger regarding females $(174.61 \pm 7.34 \mathrm{~cm}$ for males, $160 \pm 5.45 \mathrm{~cm}$ for females) and nearly equal to AbdelMalek et al. (1990) regarding male and taller regarding females $(172.8 \pm 7.2 \mathrm{~cm}$ for males, $158.9 \pm 5.37 \mathrm{~cm}$ for females). This may be endorsed by a wide range of age included in this study and sociodemographic characteristics related to Sharkia governorate population.

The results of this study showed that there was correlation (positive) between participant's stature and all handprint parameters proved by correlation coefficient between stature and different hand and phalangeal print measurements in the participants.

These results came similar to results found by Abdel-Malek et al. (1990) and others achieved the same result including Krishan and Sharma (2007), Rastogi and Paulis (2015).

Phalangeal lengths have a positive with significant correlation with the stature, this

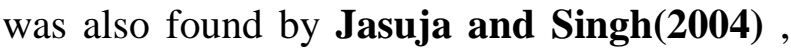
who studied all the three phalanges of each finger of Punjabi Jat Sikhs (in India) and Shintaku and Furuya (1990) who reported for Japanese women a similar correlation of proximal phalange and stature .Using hand and phalangeal print measurements for estimation of stature could be explained from the previous studies which proved that stature can be predicted constantly from the arm span (Quanjer et al.,2014).

There was marked correlation in positive mode ( $\mathrm{p}<0.05)$ among men and women regarding stature and all handprint parameters showed by correlation coefficient between stature and different handprint parameters in the study population. Hand length in both the genders expressed larger coefficients of correlation with stature, for estimation of stature; hand length emerged as a respectful tool. These results were in concordance with Aboul-Hagag et al.(2011) and Ishak et al. (2012) who also found that hand length showed higher correlation s with stature.

As far as we know, this is the first research to estimate sex in Sharkia governorate, Egypt, from handprint and phalangeal print measurements taken by scanner. While AboulHagag et al. (2011) studied hand dimensions as index and ring finger length ratio for sex estimation in Upper Egypt.

The current research showed considered sexual dimorphism in the measurements of hand prints in the Sharkia governorate population and handprint breadth was the most dimorphic variable, this came in concordance with Kanchan and Rastogi (2009) and Krishan et al.(2016) both similarly found that hand breadth was the most dimorphic parameter in a North and South Indian population respectively.

In this study, handprint breadth and length were the most sexually dimorphic variable that was in line with Ishak et al. (2012) who found in Western Australian population the hand breadth and handprint length were the most dimorphic and that was expected because the fleshed hand is relatively presented with the 
than males as women generally have a higher body fat percentage than men(Freedman et al.,1990).

This study showed that the handprint breadth, distal phalangeal print of thumb finger (thumb b), ring finger and handprint length are more sexually dimorphic in comparison to other fingers.

A total of two measurements were selected by the discriminant crossvalidation test: handprint breadth and thumb b; sex can be estimated from this equation:

Sex $=-18.096+1.163 *$ Hand breadth + 2.458* Thumb b.

If the result above or equal to 0.0255 this indicates that the handprint belongs to men and if below 0.0255 predicts that the handprint belongs to women, sex classification by Cross-validation accuracy was $86.6 \%$ with a sex bias of $-0.8 \%$. Determination of sex from hand print parameters (section level $\geq 0.0255$ distinguish men), (section level $<0.0255$ distinguish women) with accuracy $86.6 \%$ in the population of Sharkia governorate.

By using jackknife approach, the results were crossed validated to evaluate the predictive error and the discriminant analysis of the handprint data. The overfitting of this data was avoided by predicting group affiliation using functions based on samples that did not include the individuals that were being classified (Kovarovic et al., 2011 and Cardini et al.,2009). This is in concordance with what was found in Kanchan and Rastogi (2009) and Krishan et al.(2016), both of which presented results showing classifications accuracies above $85 \%$ by using hand breadth but handprint length instead of distal phalangeal print of thumb finger(thumb b).

In relation to previous research, Jowaheer and Agnihotria (2011) and also Ishak et al.(2012) demonstrated that hand breadth and length can be used to estimate sex with an expected accuracy of above $90 \%$. This result concurs with the findings of the present study, specifically that it is possible accuracy based on the statistical analysis of anthropometric hand variables.

The posterior probability of a random event or an uncertain proposition is: "The conditional probability that is assigned after the relevant evidence or background is taken into account"(Donavan and Mickey, 2019). The posterior probability of that study showed that men were correctly classified at above $88.6 \%$ certainty and that women were correctly classified at above $85 \%$ and no single individual was classified at less than $40 \%$ and that was similar to Case and Ross (2007) who documented that human phalanges are sexually dimorphic and can be used to classify sex at $74.8 \%$ and even $81 \%$ accuracy.

Sexual dimorphism is expressed as absolute differences in size, shape and behavior in various studies which result from hormonal changes around puberty (Nikitovic, 2018). This explain why men and women taking different growth pathways, with women undergoing puberty earlier and stop growing at an earlier age than men (Winkler et al., 2015).

\section{CONCLUSIONS}

This study shows that there is a significant positive correlation between participant's stature and handprint parameters proved by student $-\mathrm{t}$ - test and correlation coefficient and also shows that handprint and phalangeal length print measurements were highly reliable for the estimation of stature in forensic application. The current study has defined a base for the estimation of sex in Sharkia governorate population and the expected sex classification accuracy for handprints is $86.6 \%$. An equation for that was concluded.

\section{RECOMMENDATIONS}

Further studies over larger number of populations to emphasize the current study results to increase the number of Egyptian population studied, and to determine whether age and other parameters, have an effect. Also, wider researches are recommended to assess their applicability in forensic practice. 


\section{REFERENCES}

Abdel-Malek, A. K.; Ahmed, A. M.; El Sharkawi, S. A. E. A. and El, N. A. E. M. A. (1990): Prediction of stature from hand measurements. Forensic science international, 46(3): 181-187.

Aboul-Hagag, K. E.; Mohamed, S. A.; Hilal, M. A. and Mohamed, E. A. (2011): Determination of sex from hand dimensions and index/ring finger length ratio in Upper Egyptians. Egyptian Journal of Forensic Sciences, 1(2): 80-86.

Ahemad, N. and R. Purkait (2011): Estimation of stature from hand impression: a nonconventional approach. Journal of forensic sciences56 (3): 706-709.

Cardini, A.; Nagorsen, D.; O'Higgins, P.; Polly, P. D.; Thorington Jr, R. W. and Tongiorgi, P. (2009): Detecting biological distinctiveness using geometric morphometrics: an example case from the Vancouver Island marmot. Ethology Ecology \& Evolution, 21(3-4):209-223.

Case, D. T. and Ross, A. H. (2007): Sex determination from hand and foot bone lengths. Journal of forensic sciences, 52(2): 264-270.

Colman, K. L.; Janssen, M. C. L.; Stull, K. E.; van Rijn, R. R.; Oostra, R. J.; de Boer, H. H. and van der Merwe, A. E. (2018): Dutch population specific sex estimation formulae using the proximal femur. Forensic science international, 286: 268.e1-268.e8

Donovan, T. M. and Mickey, R. M. (2019): Bayesian interference In: Bayesian Statistics for Beginners: A Step-by-step Approach. Oxford University Press; PP: 44.

Freedman, D. S.; Jacobsen, S. J.; Barboriak, J. J.; Sobocinski, K. A.; Anderson, A. J.; Kissebah, A. H., ... and Gruchow, H. W. (1990): Body fat distribution and male/female differences in lipids and lipoproteins. Circulation, 81(5), 1498-1506.

Ghasemi, A. and Zahediasl, S. (2012):

Normality tests for statistical analysis: a guide for non-statisticians.International journal of endocrinology and metabolism, 10(2), 486.

Habib, S. R. and Kamal, N. N. (2010): Stature estimation from hand and phalanges lengths of Egyptians. Journal of Forensic and Legal
Medicine, 17(3): 156-160.

Ishak, N. I.; Hemy, N. and Franklin, D. (2012): Estimation of stature from hand and handprint dimensions in a Western Australian population. Forensic science international, 216(1-3): 199-e1. Jasuja, O. P. and Singh, G. (2004): Estimation of stature from hand and phalange length. JIAFM, 26(3): 0971-0973.

Jowaheer, V. and Agnihotri, A. K. (2011): Sex identification on the basis of hand and foot measurements in Indo-Mauritian population-a model based approach. Journal of forensic and legal medicine, 18(4): 173-176.

Kanchan, T. and Rastogi, P. (2009): Sex determination from hand dimensions of North and South Indians. Journal of forensic sciences, 54(3):546-550.

Komar, D. A. and Buikstra, J. E. (2008): Crime investigation In: Forensic anthropology: contemporary theory and practice. Oxford University Press, USA; pp: $1-2$.

Kovarovic, K.; Aiello, L. C.; Cardini, A. and Lockwood, C. A. (2011): Discriminant function analyses in archaeology: are classification rates too good to be true? Journal of Archaeological Science, 38(11): 3006-3018.

Krishan, K. and Sharma, A. (2007): Estimation of stature from dimensions of hands and feet in a North Indian population. Journal of forensic and legal medicine, 14(6): 327-332.

Krishan, K.; Chatterjee, P. M.; Kanchan, T.; Kaur, S.; Baryah, N. and Singh, R. K. (2016): A review of sex estimation techniques during examination of skeletal remains in forensic anthropology casework. Forensic science international, 261:165.e1-165.e8

Kumar P, Shahnawaz, K. and Varma, G. (2014): Study of estimation of stature by the length of femur. Journal of Evolution of Medical and Dental Sciences; 3(12):31663172.

Nagwanshi, K. K. (2019). Cyber-Forensic Review of Human Footprint and Gait for Personal Identification: IAENG International Journal of Computer Science, 46(4). 
Nikitovic, D. (2018): Sexual dimorphism (humans).In: The International Encyclopedia of Biological Anthropology, Wenda trevathan (eds), John wiley and Sons Inc, pp: 1-4.

Quanjer, P. H.; Capderou, A.; Mazicioglu, M. M.; Aggarwal, A. N.; Banik, S. D.;

Popovic, S.; ... and Zelter, M. (2014): Allage relationship between arm span and height in different ethnic groups. European Respiratory Journal, 44(4), 905-912.

Oura, P.; Karppinen, J.; Niinimäki, J. and Junno, J. A. (2018): Sex estimation from dimensions of the fourth lumbar vertebra in Northern Finns of 20, 30, and 46 years of age. Forensic science international, 290: 350.e1-350.e6.

Ozaslan, A.; Karadayi, B.; Kolusayin, M. O.; Kaya, A. and Afsin, H. (2012):

Predictive role of hand and foot dimensions in stature estimation. Rom J Leg Med, 20(1):41-46.

Paulis, M. G. (2015): Estimation of stature from handprint dimensions in Egyptian population. Journal of forensic and legal medicine, 34: 55-61.

Rastogi, P.; Nagesh, K. R. and Yoganarasimha, K. (2008): Estimation of stature from hand dimensions of north and south Indians. Legal medicine, 10(4):185189.

Reel, S.; Rouse, S.; Obe, W. V. and Doherty, P. (2012): Estimation of stature from static and dynamic footprints. Forensic science international, 219(1-3): 283.e1283.e5.

Sanli, S. G.; Kizilkanat, E. D.; Boyan, N.; Ozsahin, E. T.; Bozkir, M. G.; Soames, R.; Erol, H. and Oguz, O. (2005): Stature estimation based on hand length and foot length. Clinical Anatomy: The Official Journal of the American Association of Clinical Anatomists and the British Association of Clinical Anatomists, 18(8):589-596.

Shintaku, K. and Furuya, Y. (1990): Estimation of stature based on the proximal phalangeal length of Japanese women's hands. Journal of UOEH (University of Occupational \& Environmental Health), 12(2): 215-219.
Tang, J.; Chen, R. and Lai, X. (2012):

Stature estimation from hand dimensions in a Han population of Southern China. Journal of forensic sciences, 57(6): 1541-1544.

Winkler, T. W., Justice, A. E., Graff, M., Barata, L., Feitosa, M. F., Chu, S., ... \& Lu, Y. (2015): The influence of age and sex on genetic associations with adult body size and shape: a large-scale genome-wide interaction study. PLoS genetics, 11(10). 
تحديد الطول والجنس من قياسات بصمة اليد في سكان محافظة الثرقية

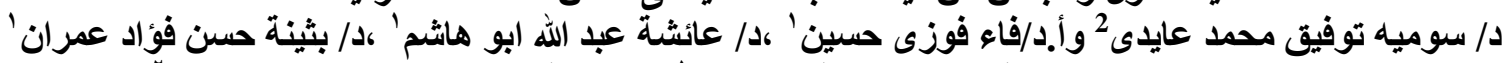

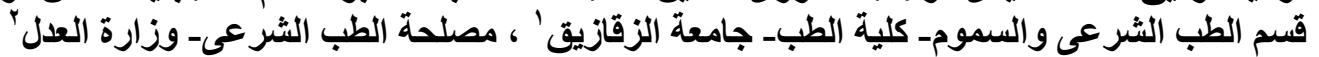

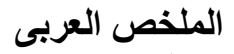

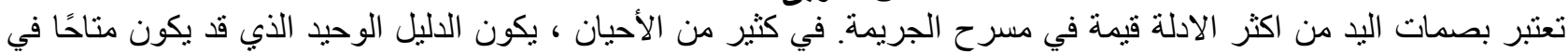

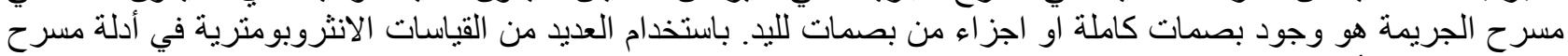

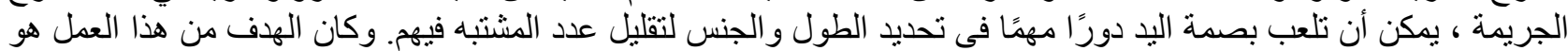

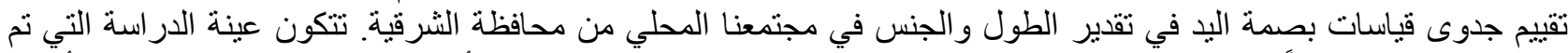

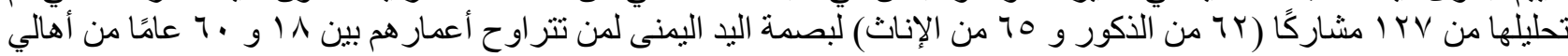

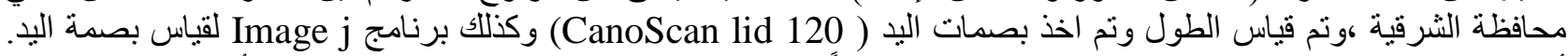

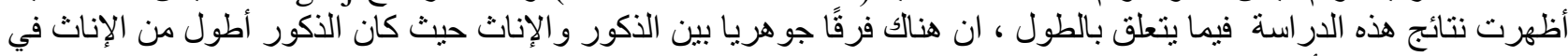

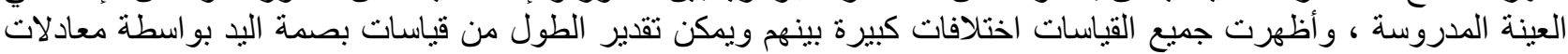

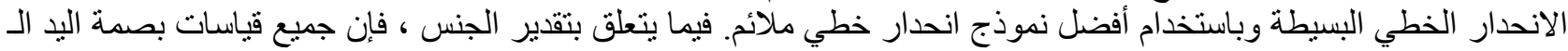

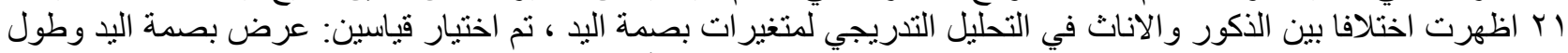

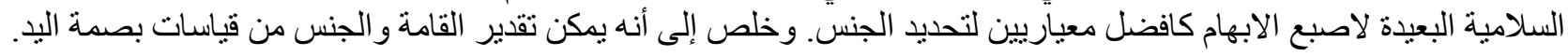

